\section{HemoShear's human touch}

\section{By Kai-Jye Lou, Senior Writer}

HemoShear LLC's October announcement that a new application of its in vitro tissue modeling platform to tumors reproduces the human response to cisplatin means the company could soon tap into business from drug developers working in oncology_an area the biotech had not yet broached.

Since its launch in 2008, HemoShear has been engaging with client companies as well as partners from academia and the public sector to develop in vitro models of human tissue and organ systems to support drug discovery and understand disease biology. Its in vitro models include $3 \mathrm{D}$ culture systems and incorporate elements of in vivo physiology such as hemodynamic forces, multilayer biological transport processes and cell-to-cell communication.

The idea is that cells cultured in environments with these elements will behave as they would in vivo and provide readouts more reliable and relevant to human biology than those from traditional in vitro and animal model systems. Standard in vitro models usually involve cell or tissue monolayers cultured under static conditions, which often behave and respond differently than in vivo microenvironments.

The company's study on cisplatin was funded by a Small Business Innovation Research (SBIR) contract from the National Cancer Institute of almost $\$ 300,000$ to develop models that recapitulate the tumor microenvironment across a wide range of cancers. One of these models reproduced the structure, biology and signaling pathways of human non-small cell lung cancer (NSCLC) and closely matched the human response to cisplatin, whereas the same dose in mice and standard cell culture systems did not.

The study represents the company's first foray into tumor models, and cofounder, CSO and VP Brett Blackman told SciBX that the company plans to expand the repertoire of in vitro systems to cover other tissues and disease areas including the blood brain barrier, lymph nodes and some undisclosed rare diseases.

Until now, the focus has been on vascular systems and the liver. The company has created 10 models in those areas and has shown that they can be used for studying drug metabolism, performing toxicity screening and recapitulating pathological states such as atherosclerosis, diabetes and hepatosteatosis. These models recapitulate hemodynamic parameters, such as pulsatile blood flow and shear stress, and transportation of oxygen and nutrients across semipermeable membranes and multiple layers of cells.

According to Zhu Chen, a principal scientist at Merck \& Co. Inc., HemoShear's platform recapitulates human physiology more faithfully than standard cell-based systems. "As blood rheology and hemodynamics are an important component of cardiovascular physiology, the traditional static cell culture approach is very limited in translational research in drug discovery and development in cardiovascular diseases," he said.

He added that because the platform can simulate hemodynamic characteristics of human vascular beds in different disease states, it supports translational studies for both target biology in disease settings and interrogation of candidate compounds with disease-relevant readouts.

Last year, researchers at HemoShear and Merck published data that suggested atrial fibrillation-induced hemodynamic changes could enhance the thrombotic potential of the endothelium in the left atrial appendage of the heart. ${ }^{1}$ "We likely would not have reached similar conclusions if studies were conducted in standard cell culture systems as they lack the critical component of atrial fibrillation-like hemodynamics and are thus less pathologically relevant," said Chen.

Other companies with in vitro systems that mimic in vivo human biology include the microfluidics-based organ-on-a-chip models being developed by Emulate Inc., Mimetas B.V. and TissUse GmbH. ${ }^{2}$

Blackman said that HemoShear differentiates itself from competitors by complementing its models with its computational biology assets. Those assets include a suite of tools for pathway analytics and interpretation of functional and transcriptomics data and access to a database of curated biological data.

The company's Pharmacological Human Response Databases contain information on vascular and liver transcriptional responses at the gene, pathway and systems levels for the more than 200 compounds from over 30 drug classes that it has evaluated.

"HemoShear's Pharmacological Human Response Databases are unique in that they were developed using human relevant healthy and disease tissue conditions, not cell lines or other traditional, nonhuman models," said Blackman.

HemoShear populates its databases with results generated from its collaborations, projects under government grants and contracts, internal $\mathrm{R} \& \mathrm{D}$ and data from the public domain.

To date, the company's in vitro models have been evaluated in collaboration with researchers from over 10 companies, including Amgen Inc., Eli Lilly and Co., Johnson \& Johnson's Janssen R\&D unit and Merck. As a consequence, Blackman said, there are substantial data available on the use of HemoShear's models across a broad range of settings. 
He added that the company's models have been used in studies to validate the safety of various marketed drugs and to retroactively recapitulate adverse effects that resulted in black-box warnings and market withdrawals.

\section{Custom models}

HemoShear's business model is based on creating custom tissue and organ models and integrating its platform capabilities with disease and drug discovery programs at client companies.

The collaborations typically fall into three categories. The first type is a discovery collaboration, in which HemoShear uses its models, knowledge base and tools to help discover targets and therapeutics in the client's disease area. According to Blackman, none of these collaborations has been established long enough for any of the compounds evaluated to have reached the market.

The second category is platform collaborations, in which HemoShear develops model systems that incorporate the necessary biological elements to help a client test its platform technology.

Finally, according to Blackman, HemoShear also does "tissue and disease model collaborations," in which it works with a partner to develop new systems that can drive model development in areas that lack good translational models. "An example would be the neurovascular system," said Blackman. "The ability to create a bona fide neurovascular system in vitro could drive the development of better blood brain barrier models as well as new models for Alzheimer's disease and multiple sclerosis."

HemoShear owns the exclusive rights to any new IP that relates to improvements and modifications to the company's core IP, proprietary biorepository and related methods, and knowledge databases in computational biology. Blackman said that quite often new IP is developed over the course of a collaboration that is not an enhancement of HemoShear's own technology. In those cases, the IP is jointly owned.

Ownership of data and models generated under collaborations vary depending on the terms of a deal, but Blackman noted that the majority of the company's current data and models are unencumbered by previous agreements.

"Clearly pharma will want exclusive rights when there is an important competitive advantage, but we also have models that have been developed in prior collaborations that can be used in current and future partnerships," he told SciBX.

Blackman said that the most recent data HemoShear co-published with researchers from J\&J's Janssen R\&D unit provide an example of the company's approach to data generated under collaborations.

HemoShear presented data at the American College of Rheumatology meeting this week suggesting that anti-IL- 6 therapies for rheumatoid arthritis (RA), such as sirukumab from J\&J and Actemra tocilizumab from Roche's Genentech Inc. unit, could have the added benefit of improving vascular health over RA therapies that target tumor necrosis factor- $\alpha$ (TNF- $\alpha$ ) and JAK kinase (JAK) pathways. HemoShear used its human vascular tissue system models in the study.
HemoShear said in a press release that the results "lay the groundwork to explore potential new biomarkers for diagnosing RA patients with increased risk for cardiovascular disease and to discover new therapeutic targets for RA and cardiovascular disease."

$\mathrm{RA}$ is an independent risk factor for the development of atherosclerosis, a vascular disease with a strong inflammatory component. ${ }^{3}$ J\&J has sirukumab in Phase III testing to treat RA

Chugai Pharmaceutical Co. Ltd., Roche and Genentech already market Actemra for the indication.

Blackman said that the majority of HemoShear's collaborations have involved the partner providing the company with $\mathrm{R} \& \mathrm{D}$ funding.

He added, "We envision future collaborations in which we will receive milestone payments and royalties from our partners. To date, we have not negotiated these terms, as HemoShear's work up to this point has focused on advancing our platform and pilot stage and proof-of-concept programs. In 2014, we initiated discussions with pharma and biotech companies for collaborations that include potential milestones, royalties and success payments."

Its most recently announced industry collaboration is with Medivir AB. In April, HemoShear said in a press release that it will use its human liver system to characterize the mechanisms of action and liver tissue effects of undisclosed drug classes. HemoShear said that Medivir will use data generated from the study for discovery research in undisclosed strategic areas and that future work under the deal may include specific drug programs.

HemoShear has raised $\$ 22$ million in private financing over the past 6 years. The company also has received about $\$ 10$ million in funding from the NIH and other federal and state sources. Blackman said that the company is seeking new investors.

Lou, K.-J. SciBX 7(45); doi:10.1038/scibx.2014.1311

Published online Nov. 20, 2014

\section{REFERENCES}

1. Wamhoff, B.R. et al. Arterioscler. Thromb. Vasc. Biol. 33, A73 (2013)

2. Schaffer, C. BioCentury 15; Sept. 22, 2014

3. Del Rincón, I. et al. Atherosclerosis 195, 354-360 (2007)

\section{COMPANIES AND INSTITUTIONS MENTIONED}

American College of Rheumatology, Atlanta, $\mathrm{Ga}$.

Amgen Inc. (NASDAQ:AMGN), Thousand Oaks, Calif.

Chugai Pharmaceutical Co. Ltd. (Tokyo:4519), Tokyo, Japan

Eli Lilly and Co. (NYSE:LLY), Indianapolis, Ind.

Emulate Inc., Cambridge, Mass.

Genentech Inc., South San Francisco, Calif.

HemoShear LLC, Charlottesville, Va.

Johnson \& Johnson (NYSE:JNJ), New Brunswick, N.J.

Medivir AB (SSE:MVIR B), Huddinge, Sweden

Merck \& Co. Inc. (NYSE:MRK), Whitehouse Station, N.J.

Mimetas B.V., Leiden, the Netherlands

National Cancer Institute, Bethesda, Md.

National Institutes of Health, Bethesda, Md.

Roche (SIX:ROG; OTCQX:RHHBY), Basel, Switzerland

TissUse GmbH, Berlin, Germany 